\title{
The Role of the Access Point in Wi-Fi Networks with Selfish Nodes
}

\author{
Ilenia Tinnirello \\ Laura Giarrè \\ Giovanni Neglia
}

\begin{abstract}
In Wi-Fi networks, mobile nodes compete for accessing the shared channel by means of a random access protocol called Distributed Coordination Function (DCF), which is long term fair. But recent drivers allow users to configure protocol parameters differently from their standard values in order to break the protocol fairness and obtain a larger share of the available bandwidth at the expense of other users. This motivates a game theoretical analysis of DCF.

Previous studies have already modeled access to a shared wireless channel in terms of non-cooperative games among the nodes, but they have mainly considered ad hoc mode operation. In this paper we consider the role of the Access Point (AP) in infrastructure mode operation, for mitigating or discouraging such selfish behaviors. Solving a mechanism design problem, we use the AP as a network coordinator, for encouraging node strategies which maximize a global utility function. We analyze both unidirectional and bidirectional (uplink and downlink) traffic scenarios. It is well known that nodes selfishness jeopardize performance, but we show that simple changes to AP operation can let the system achieve optimal performance in spite of nodes selfishness. Instead for the bidirectional scenario the legacy behaviour of an AP is sufficient to guarantee quasi optimal performance.
\end{abstract}

\section{INTRODUCTION}

The problem of resource sharing in IEEE 802.11 [1] networks is addressed by the Medium Access Protocol (MAC) according to the carrier sense multiple access with collision avoidance (CSMA/CA) paradigm. The protocol relies on random deferments of packet transmissions, distributely computed by each contending station, in order to avoid synchronized accesses to the channel. In fact, simultaneous transmissions interfere and result in the loss of the transmitted frames.

The distributed protocol is in principle fair, because in long term each station receives the same number of access opportunities. Nevertheless it has been observed that cards produced by different vendors (and certified by the WiFi alliance [2]) experience different performance. In many cases, such different behaviors have been recognized as a consequence of malicious settings of the MAC parameters [3], whose configuration is often easily available to end users thanks to open-source drivers. Since the MAC protocol regulates the resource repartition among multiple users in a distributed manner, selfish nodes gain more resources at the expense of other users.

ilenia.tinnirello@tti.unipa.it, D.I.E.E.T. (Dipartimento di Ingegneria Elettrica, Elettronica e delle Telecomunicazioni),Universitá degli Studi di Palermo, viale delle scienze, 90128 Palermo, Italy

giarre@unipa.it, D.I.A.S. (Dipartimento di Ingegneria dell'Automazione e dei Sistemi), Universitá degli Studi di Palermo, viale delle scienze, 90128 Palermo, Italy

giovanni.neglia@inria.fr, Équipe Projet INRIA Maestro, 2004 route des Lucioles, 06902 Sophia Antipolis, France
Resource sharing in Wi-Fi networks have often been modeled in terms of non-cooperative game among the stations. Most of these approaches assume that the station utility function is expressed by the node throughput or related to this performance figure. In [5], it has been shown that a utility function equal to the throughput may lead to a Nash Equilibrium (NE) in which stations do not defer their transmission anymore. This situation creates a resource collapse, because all stations transmit simultaneously thus destroying all packet transmissions. More complex utility functions combining throughput and costs related to collision rates [5], [6] or to energy consumptions [7] lead to different equilibria, but at the same time they appear less natural. Distributed mechanisms to drive the the system to socially good operation point have also been proposed [5].

In relation to existing literature our work has two main novel contributions. First, we explicitly consider infrastructure network scenarios, in which $n$ greedy mobile nodes compete for accessing the Internet through a common Access Point (AP). While each node tries to maximize each utility, the access point may be configured by the network administrator in order to counteract nodes selfishness. We show that this approach is promising, in fact we are able to design simple mechanisms that can be deployed at the AP and can guarantee optimal global performance. The second main contribution is that we do not only study the unidirectional scenario, where users simply need to upload some information and so want to maximize their uploading rate, but we consider also a bidirectional scenario in which user uplink traffic generates downlink traffic from the Internet. In this case the user is interested in maximizing at the same time its uploading and downloading rate.

The rest of the paper is organized as follows. Section II briefly summarizes the DCF operations and the node strategy model; section III proposes some punishment strategies for imposing collision costs in case of unidirectional traffic; section ?? analyzes the case of coexistence between uplink and downlink streams; finally, some conclusions are drawn in section $\mathrm{V}$. Extended results are provided in a companion technical report [?].

\section{DCF OPERATIONS AND MODELS}

We assume that the reader is familiar with the IEEE 802.11 Distributed Coordination Function (DCF) and its performance evaluation. Thus, we briefly summarize the protocol operation and the modeling approaches used for our game formulation.

In DCF, a station with a new data packet to be transmitted monitors channel activity. If the channel is idle for a period 
called Distributed InterFrame Space (DIFS), the station transmits. The receiving station signals the successful reception by replying with an acknowledgment frame (ACK). The ACK is transmitted at the end of the data packet reception, and within a period of time called Short InterFrame Space (SIFS). The SIFS duration is shorter than the DIFS one, to impede that other stations trying to access the channel may interfere with the ACK transmission. If the transmitting station does not receive the ACK within a specified ACK timeout, it reschedules the packet transmission after a random interval of time, using the backoff rules described in what follows. Stations revealing the corrupted frame on the channel wait for an Extended Interframe Space (EIFS) before resuming the channel access procedure.

DCF employs a technique called collision avoidance to reduce the probability that two or more competing stations simultaneously transmit and hence cause packet corruption. Whenever the channel is sensed busy, the station continues to monitor the channel until it is idle for a DIFS. At this point, rather than immediately transmitting, the station schedules the packet transmission after a random time interval called backoff ${ }^{1]}$ For efficiency reasons, the backoff interval is expressed as an integer number of backoff slots. The number of waiting slots is called backoff counter and is uniformly chosen in the range $(0, w-1)$, where $w$ is the contention window. The backoff counter is decremented as long as the channel is sensed idle, frozen when a transmission is detected on the channel, and reactivated when the channel is again sensed idle for more than a DIFS. The station transmits when the backoff counter reaches 0 . The range in which the counter is extracted (i.e. $w$ ) is an adaptive parameter, which follows a truncated exponential increment law (doubling from $C W_{\text {min }}$ up to $C W_{\max }$ ), according to the number of consecutive failed transmissions.

Although DCF is based on the backoff process previously described, it has been shown [9] that it can be accurately modeled as a persistent slotted access protocol. When all stations are permanently in contention, that is when they all have always at least one frame in the transmission buffer, the interval between the end of activity on the channel and the next access is always equivalent to a entire number of idle backoff slots. Considering a nonuniform scale for slots, in which each slot represents either an idle backoff slot or an activity period plus the final DIFS time, the behavior of the protocol can be summarized by a single parameter $\tau$, representing the probability that a tagged station accesses the channel during a generic channel slot:

$$
\tau=\frac{1}{1+E[b]}
$$

where $E[b]$ is the average backoff interval, that can be derived as a function of the average contention window, which in turns depend on the collision probability. Specifically, the equivalent channel access probability $\tau$ of a legacy station

\footnotetext{
${ }^{1}$ A random backoff is also extracted between consecutive packet transmissions.
}

experiencing a collision probability $p$ is given by:

$$
\tau=f(p)= \begin{cases}\frac{2\left(1-p^{R+1}\right)}{1-p^{R+1}+(1-p) \sum_{i=0}^{R} p^{i} W(i)} & 0 \leq p<1 \\ \frac{2(R+1)}{1+\sum_{i=0}^{R} W(i)} & p=1\end{cases}
$$

where $R$ is the retry limit employed in the network and $W(i)$ is the contention window at the $i-t h$ retry stage (i.e. $W(i)=$ $\left.\min \left\{2^{i} C W_{\min }, C W_{\max }\right\}\right)$.

A very obvious way for differentiating station performance is the adoption of nonuniform contention window settings. In fact, stations employing lower contention windows will probabilistically expire their backoff counter more often and will transmit more packets than the competing stations. Thus, we assume that the strategy of each selfish user $i$ is represented by the selection of the contention window ranges or equivalently its channel access probability.

\section{UNIDIRECTIONAL TRAFFIC SCENARIO}

In this section we consider the application scenario referred in most of the related literature, according to which only uplink data streams are present. In particular a fixed number of mobile stations (say it $n$ ) transmit to a single Access Point (AP). It is quite natural to consider that a selfish mobile station would like to maximize its uploading rate. For this purpose we assume that each station $i$ can arbitrarily set its channel access probability $\tau_{i}$. The set of strategies is then $[0,1]^{n}$ and we define an outcome of the game a set of strategies taken by the players, then a vector $\boldsymbol{\tau}=\left(\tau_{1}, \tau_{2}, \cdots, \tau_{n}\right) \in[0,1]^{n}$. Being that in our case all the players are homogeneous, the equilibria we are going to define are invariant to player permutations. For this reason we define two outcomes $\tau^{a}$ and $\tau^{b}$ equivalent if they can be obtained one from the other through an opportune permutation of the indexes and we write $\tau^{a} \sim \tau^{b}$. We denote a class of equivalent outcomes as $\{\tilde{\boldsymbol{\tau}}\}$, where $\tilde{\boldsymbol{\tau}}$ is an ordered vector, i.e. a vector with increasing component $\left(\tilde{\tau}_{1} \leq \tilde{\tau}_{2} \leq \cdots \tilde{\tau}_{n}\right)$. If $A$ is a set of ordered vectors, then $\{A\}$ denotes the union of the classes of equivalence of the vectors in $A$.

Station $i$ experiences collisions during its own transmissions, when at least one of the competing nodes transmits in the same slot. This happens with probability $p_{i}=1-$ $\prod_{j \neq i}\left(1-\tau_{j}\right)$. Note that the product $P_{i d l e}=\left(1-\tau_{i}\right)\left(1-p_{i}\right)$ is the probability that no station accesses the channel in a given slot and does not depend on the index $i=1,2, \cdots n$. Following [8], we can express the uplink throughput $S_{u}^{i}$ for the $i$-th station as:

$$
S_{u}^{i}=\frac{\tau_{i}\left(1-p_{i}\right) P}{P_{i d l e} \sigma+\left[1-P_{i d l e}\right] T}
$$

where $P$ is the frame payload which is assumed to be fixed, while $\sigma$ and $T$ are, respectively, the empty and the busy slot duration ${ }^{2}$, so that the denominator is the expected slot duration $\left(E\left[T_{\text {slot }}\right]\right)$.

\footnotetext{
${ }^{2} \mathrm{We}$ are implicitly considering a basic access scheme, with EIFS=ACK_Timeout +DIFS, which corresponds to have a fixed busy slot duration in both the cases of successful transmission and collision.
} 
We define the utility function $J_{U N}^{i}$ for the mobile station $i$ equal to its uploading rate, then

$$
J_{U N}^{i}=S_{u}^{i} .
$$

\section{A. Nash equilibria}

The following remark will be useful to characterizes Nash equilibria and identify Pareto optimal outcomes.

Remark 3.1: In general player $i$ utility is a function of the whole set of strategies $(\boldsymbol{\tau})$, but it is constant and equal to 0 if a) $p_{i}=1$, i.e. if at least one of the other player is transmitting with probability $1\left(\exists j \mid \tau_{j}=1\right)$, or if b) $\tau_{i}=0$. In all the other cases $J_{U N}^{i}$ is strictly increasing in user $i$ strategy $\tau_{i}$ and strictly decreasing in other users strategies. As a consequence, if $\tau \in[0,1)^{n}$, then $J_{U N}^{i}<J_{j}$ if and only if $\tau_{i}<\tau_{j}$

In [5] the authors study the Nash equilibria of this game and they prove the following result that we show here for the sake of completeness.

Proposition 3.1: The Nash equilibria of the game described above are all and only the vectors of strategies $\tau$, such that $\exists i \in 1,2, \cdots n \mid \tau_{i}=1$.

Proof: The result is an immediate consequence of remark 3.1.

We observe that we can distinguish two different set of Nash equilibria. If there is only a single station $i$ transmitting with probability 1 , this station achieves an uplink rate equal to $S_{u}^{i}=P\left(1-p_{i}\right) / T=C_{u}\left(1-p_{i}\right)$, where $C_{u}$ is the uplink capacity. All the other stations are not able to transmit $S_{u}^{j}=0$ for $j \neq i$. This set of strategies is $\{(\boldsymbol{x}, 1), \boldsymbol{x} \in$ $\left.[0,1)^{n-1}\right\}$. In particular for $(0,0, \cdots, 0,1)$ the single station transmitting is able to achieve the maximum throughput $C_{u}$. If there are two or more stations transmitting with probability 1 , then the channel is entirely wasted because of collisions and $S_{u}^{h}=0, \forall h$. This set of strategies is then $\{(\boldsymbol{x}, 1,1), \boldsymbol{x} \in$ $\left.[0,1]^{n-2}\right\}$. Performance at these Nash Equilibria are very poor. In what follows we design a different game, changing the behaviour of the Access Point, in order to improve global performance.

\section{B. Mechanism Design}

In order to improve the performance achieved in presence of selfish mobile stations, we introduce some incentive mechanisms by adding new simple functionalities to the Access Point. As a preliminary step, we need to identify desirable outcomes from the global point of view. A natural choice is to look at outcomes that maximize the global utility, i.e. the aggregate uplink rate $\sum_{i} J_{U N}^{i}=\sum_{i} S_{u}^{i}$. It is easy to derive that, given the probabilistic access protocol, the maximum uplink rate can be achieved only when there is a single station transmitting with probability 1 and all the other stations are silent. These are the NE outcomes of the type $\{(0,0, \cdots 0,1)\}$. We observe that they are Pareto optimal outcomes. We remember that a Pareto optimal outcome is one such that no-one could be made better off by changing the vector of strategies without making someone else worse off. At the same time, the outcomes $\{(0,0, \cdots 0,1)\}$ appear to be extremely unfair. This motivates us to look for outcomes, that maximize the uplink rate under a fairness constraint. We want then to consider as social utility the following function:

$$
J_{S}=\min _{i=1, \cdots n} J_{U N}^{i}
$$

Proposition 3.2: There is a unique outcome that maximizes $J_{U N}^{S}$ and such outcome is homogeneous, i.e. $\tau_{U N}^{*}=$ $\left(\tau_{U N}^{*}, \tau_{U N}^{*}, \ldots \tau_{U N}^{*}\right)$, and Pareto optimal.

Proof: First we observe that the social utility is null $\left(J_{U N}^{S}=0\right)$ for the sets of strategies $\left\{(0, \boldsymbol{x}), \boldsymbol{x} \in[0,1]^{n-1}\right\}$ and $\left\{(\boldsymbol{x}, 1), \boldsymbol{x} \in[0,1]^{n-1}\right\}$, while it is strictly positive otherwise, i.e. for the set of strategies $A=\left\{\boldsymbol{x}, \boldsymbol{x} \in(0,1)^{n}\right\}$ (every player has a positive uploading rate). We can then restrict our attention to $A$.

Let $\tau$ be a non-homogeneous outcome in $A$, we prove that social utility cannot be maximum for $\tau$. Without loss of generality we consider that $\tau$ is an ordered vector, then $1>\tau_{n} \geq \tau_{n-1} \geq \cdots \tau_{1}>0$ with $\tau_{n}>\tau_{1}$ and $J_{U N}^{S}(\boldsymbol{\tau})=$ $J_{1}(\boldsymbol{\tau})$. Let us consider $\tau_{n}^{\prime}$, such that $\tau_{1}<\tau_{n}^{\prime}<\tau_{n}$, the outcome $\boldsymbol{\tau}^{\prime}=\left(\tau_{1}, \tau_{2}, \cdots, \tau_{n-1}, \tau_{n}^{\prime}\right)$ has higher social utility than $\boldsymbol{\tau}$. In fact $J_{U N}^{S}\left(\boldsymbol{\tau}^{\prime}\right)=\min _{i} J_{U N}^{i}\left(\boldsymbol{\tau}^{\prime}\right)=J_{1}\left(\boldsymbol{\tau}^{\prime}\right)>$ $J_{1}(\boldsymbol{\tau})=J_{U N}^{S}(\boldsymbol{\tau})$. The second and third equalities follows from $\tau_{1}$ being the smallest element both in $\tau^{\prime}$ and in $\tau$. The inequality follows from player $n$ having reduced its access probability from $\tau_{n}$ to $\tau_{n}^{\prime}$ (see Remark 3.1.

If we focus on homogeneous outcomes, we can consider the single variable function $J_{U N}^{S}((\tau, \tau, \cdots, \tau))$. The sign of its derivative is equal to the sign of

$$
-\frac{T-\sigma}{T}(1-\tau)^{n}+n(1-\tau)-n+1
$$

This expression is positive for $\tau=0$, non positive for $\tau=1$ (in particular negative for $n>1$ ) and strictly decreasing in $\tau$. We can then observe that there is a unique point of maximum of the single variable function $J_{U N}^{S}((\tau, \tau, \cdots, \tau))$ -say it $\tau_{U N^{-}}^{*}$ and then a unique point of maximum of the function $J_{U N}^{S}(\boldsymbol{\tau})$ for $\boldsymbol{\tau}_{U N}^{*}=\left(\tau_{U N}^{*}, \tau_{U N}^{*}, \cdots, \tau_{U N}^{*}\right)$.

Finally we prove Pareto optimality. Say $\tau$ an allocation different from $\tau_{U N}^{*}$ with at least a player better off than in $\tau_{U N}^{*}$. This allocation is necessarily non-homogenous (among the homogeneous allocations every player utility is maximized at $\left.\boldsymbol{\tau}_{U N}^{*}\right)$. Let $j=\operatorname{argmin}_{h} J_{h}(\boldsymbol{\tau})$, then $J_{U N}^{S}(\boldsymbol{\tau})=J_{j}(\boldsymbol{\tau})$. From the uniqueness of $J_{U N}^{S}$ maximum, it follows that $J_{U N}^{S}(\boldsymbol{\tau})<J_{U N}^{S}\left(\boldsymbol{\tau}_{U N}^{*}\right)=J_{j}\left(\boldsymbol{\tau}_{U N}^{*}\right)$. Then player $j$ has a lower utility at $\tau$ than at $\tau_{U N}^{*}$ and $\tau_{U N}^{*}$ is a Pareto outcome.

Remark 3.2: The outcome $\tau_{U N}^{*}$ is not a Nash Equilibrium, in fact every player can increase its utility by increasing its own access probability.

In [8] it has been shown that the optimal value $\tau_{U N}^{*}$ can be approximated for $T>>\sigma$ as:

$$
\tau_{U N}^{*} \approx \frac{1}{n \sqrt{T / 2 \sigma}} .
$$


Incidentally we observe that, given this definition of the global utility, both price of anarchy and price of stability ${ }^{3}$ have infinite value for this game. In fact in all the NEs there is at least one player with throughput equal to 0 , so $J_{U N}^{S}\left(\tau_{N E}\right)=0$.

We want to design the game so that $\tau_{U N}^{*}$ is a NE. This case requires some punishment policies in order to prevent that each station $i$ accesses the channel with probability $\tau_{i}=1$. Punishment policies proposed in previous works are usually based on jamming. A jam signal is a short burst sent on the channel in order to interfere with another transmission and creating an artificial collision. The responsibility of jamming the packets is collectively carried out by all the nodes in the system in order to introduce a uniform cost for all transmissions [5].

Indeed, since we are referring to infrastructure mode operation, where an AP is present, we can design a different punishment strategy, whose responsibility is given to the AP only. Specifically, for saving energy and avoiding distributed cost assignments, we create artificial collisions by simply suppressing some ACK transmissions originated by the AP. Since the AP is the common receiver for all the stations, suppressing the ACKs from the AP side corresponds to triggering ACK timeouts at the station side, which are interpreted as collisions. We propose the following threshold scheme: if a generic station $i$ has access probability $\tau_{i}$ higher than the a given value $\bar{\tau}$, the AP drops an ACK frame transmission with probability $\min \left\{\alpha\left(\tau_{i}-\bar{\tau}\right), 1\right\}$.

In this case, the utility function $J_{U N}^{i}$ of a given station $i$ can be expressed as:

$$
J_{U N}^{i}= \begin{cases}\frac{\tau_{i}\left(1-p_{i}\right)}{E\left[T_{\text {slot }}\right]} & 0<\tau_{i}<\bar{\tau} \\ \frac{\tau_{i}\left(1-p_{i}\right)\left[1-\alpha\left(\tau_{i}-\bar{\tau}\right)\right]}{E\left[T_{\text {slot }}\right]} & \bar{\tau} \leq \tau_{i}<\bar{\tau}+1 / \alpha \\ 0 & \bar{\tau}+1 / \alpha \leq \tau_{i} \leq 1\end{cases}
$$

where $E\left[T_{\text {slot }}\right]=P_{\text {idle }} \sigma+\left[1-P_{\text {idle }}\right] T$ is equal to the denominator in equation (3) and we recall that $P_{\text {idle }}=$ $\left(1-\tau_{i}\right)\left(1-p_{i}\right)$. According to the previous expression, for $\tau_{i} \leq \bar{\tau}$ the utility function $J_{U N}^{i}$ is an increasing function of $\tau_{i}$, while for $\tau_{i} \leq \bar{\tau}$ its slope depends on the $\alpha$ setting. By selecting an $\alpha$ value which corresponds to a negative derivative for $\bar{\tau}<\tau_{i}<\bar{\tau}+1 / \alpha$, the utility function is maximized for $\tau_{i}=\bar{\tau}$.

We can then prove the following result.

Proposition 3.3: The outcome $\tau_{U N}^{*}$ is a Pareto optimal Nash equilibrium of the game, when the ACK suppression scheme indicated above is implemented with

$$
\begin{aligned}
\bar{\tau} & =\tau_{U N}^{*}, \\
\alpha & \geq \frac{1}{\tau_{U N}^{*}\left(1+\tau_{U N}^{*}\left(-1+\frac{T}{T-(T-s)\left(1-\tau_{U N}^{*}\right)^{n-1}}\right)\right)} .
\end{aligned}
$$

Proof: First we observe that $\tau_{U N}^{*}$ is a NE. In fact, whatever player we consider, say it player $i$, Remark 3.1

\footnotetext{
${ }^{3}$ We remind that the price of anarchy is the ratio between the social utility of the optimal coordinated outcome (i.e. when users are not selfish), and the utility of the worst NE. The price of stability has a similar definition but it consider the best NE rather than the worst one.
}

guarantees that for $\tau_{i}<\tau_{U N}^{*} J_{U N}^{i}$ decreases as $\tau_{i}$ decreases. For $\tau_{U N}^{*}<\tau_{i}<\tau_{U N}^{*}+1 / \alpha$ inequality $(7)$ guarantees that $J_{U N}^{i}$ decreases as $\tau_{i}$ increases until it does not reach the value 0 . For $\tau>\tau_{U N}^{*}+1 / \alpha$, the punishment strategy implies $J_{U N}^{i}=0$. Then deviating from $\tau_{U N}^{*}$ is not convenient for player $i$.

Second, $\tau_{U N}^{*}$ is still the unique point of maximum for $J_{U N}^{S}(\boldsymbol{\tau})$ because the punishment strategy can only decrease the social utility for $\tau \in[0,1]^{n}-\left[0, \tau_{U N}^{*}\right]^{n}$ and keeps it unchanged otherwise. Reasoning as in 3.2. we can conclude that the outcome $\tau_{U N}^{*}$ is Pareto optimal.

still maximizes it can be observed that the punishment strategy decreases the social utility $J_{U N}^{S}(\boldsymbol{\tau})$ for $\boldsymbol{\tau} \in$ $\left(\tau_{U N}^{*}, 1\right]^{n}$ and keeps it unchanged otherwise, so that the Proposition 3.2 holds also under the punishment strategy.

We observe that in this new game the outcomes $\{(\boldsymbol{x}, 1), \boldsymbol{x} \in$ $\left.[0,1)^{n-1}\right\}$ are no more NEs. Nevertheless the outcomes $\left\{(\boldsymbol{x}, 1,1), \boldsymbol{x} \in[0,1]^{n-2}\right\}$ are still NEs, where all user utilities are zero. Then, while the price of stability is equal to 1 (there is a NE with optimal performance), the price of anarchy of the game is still infinite. At the same time these other NEs are not Pareto optimal, so that the outcome $\tau_{U N}^{*}$ looks a more reasonable operation point.

\section{BIDIRECTIONAL TRAFFIC SCENARIO}

Nodes belonging to an infrastructure network are usually involved into two different data streams: on one side, they need to upload traffic to the AP, which is connected to external networks; on the other side, they need to download traffic from the external networks through the AP. The first data stream is usually referred as uplink data stream, while the second one is usually referred as downlink data stream. In this section, we define a utility function which takes into account both the streams and we analyze the impact of such a definition on the network equilibria.

We assume that the AP behaves as a legacy DCF station with saturated downlink traffic. Thus, it randomly defers its transmissions according to backoff counter extractions performed in the range $[0, w-1]$, where $w$ follows the updating law described in section $\Pi$.

Let again $\boldsymbol{\tau}=\left(\tau_{1}, \tau_{2}, \cdots \tau_{n}\right)$ be the vector of strategies taken by the players, i.e. by the $n$ mobile stations. We consider the AP separately because it is not a player, but simply follows legacy operation. For a given station $i$, the strategies of the competing stations can be summarized into the probability $p_{i}=1-\prod_{j \neq i}\left(1-\tau_{j}\right)$. Thus, from the station point of view, the vector strategy $\tau$ can be represented by the couple of values $\left(\tau_{i}, p_{i}\right)$. Note that $p_{i}$ simply represents the probability that no mobile station transmits in the current slot, and it is no more equal to the collision probability because it does not take into account the AP. The overall collision probability suffered by station $i$ results equal to $1-\left(1-p_{i}\right)\left(1-\tau_{A P}\right)$, where $\tau_{A P}$ is the channel access probability employed by the AP, which is function of the perceived collision probability $p_{A P}$ according to (2). We can evaluate the AP collision probability as a function of 
Fig. 1. Utility of a given station $i$, for different $p_{i}$ values, as a function of the strategy $\tau_{i}$.

Fig. 2. Station utility in case of symmetric access probability employed by all the stations.

the vector strategy $\tau$ or as a function of the a generic pair $\left(\tau_{i}, p_{i}\right)$ :

$$
p_{A P}=1-\prod_{i=1}^{n}\left(1-\tau_{i}\right)=1-\left(1-p_{i}\right)\left(1-\tau_{i}\right)
$$

Moreover, the probability that a channel slot is idle is the probability that neither the stations, nor the AP transmit on the channel, i.e. $P_{i d l e}=\left(1-p_{A P}\right)\left(1-\tau_{A P}\right)$. Assuming that the AP equally shares the downlink throughput among the stations, we can readily express the uplink throughput $S_{u}^{i}$ and the downlink throughput $S_{d}^{i}$ for the $i$-th station as:

$$
\begin{gathered}
S_{u}^{i}\left(\tau_{i}, p_{i}\right)=\frac{\tau_{i}\left(1-p_{i}\right)\left(1-\tau_{A P}\right) P}{P_{i d l e} \sigma+\left[1-P_{i d l e}\right] T} \\
S_{d}^{i}\left(\tau_{i}, p_{i}\right)=\frac{1}{n} \frac{\tau_{A P}\left(1-p_{A P}\right) P}{P_{i d l e} \sigma+\left[1-P_{i d l e}\right] T}
\end{gathered}
$$

where $p_{A P}$-and $\tau_{A P}=f\left(p_{A P}\right)$ - can be expressed as a function of the pair $\left(\tau_{i}, p_{i}\right)$. Since the downlink throughput is equal for all the stations, we can avoid the $i$ apex. We define the utility function $J_{B I}^{i}$ for the mobile station $i$ as:

$$
J_{B I}^{i}=\min \left\{S_{u}^{i}, S_{d}\right\} .
$$

The rationale of such a definition is the assumption that the station applications require bandwidth on both directions, and they cannot work whenever the throughput on a given direction (either uplink or downlink) is zero.

Figure 1 plots the utility of a given station $i$ versus its access probability $\tau_{i}$ for different values of $p_{i}$, i.e. different strategies of the other players. The frame payload $P$ has been considered equal to 1500 bytes, the other parameters have been determined considering a IEEE $802.11 \mathrm{~b}$ physical layer, with data rate equal to $11 \mathrm{Mbps}$, and an $\mathrm{ACK}$ rate of $1 \mathrm{Mbps}$. In such a scenario, by including physical preambles, MAC headers and interframe times, the $T$ duration is equal to 1667 $\mu s$ and $\sigma$ duration to $20 \mu \mathrm{s}$. From the figure, it is evident that, for each $p_{i}$, the utility is maximized for a given best response value (about 0.01 for $p=0.15$ ), which slightly decreases as $p_{i}$ grows. Figure 2 plots again the utility of a given station in case of homogeneous outcome $\tau=(\tau, \tau, \cdots \tau)$, for different number of competing mobile stations. In these curves $p_{i}=$ $\left(1-\tau_{i}\right)^{n-1}$ is not fixed, because the strategy changes are not unilateral. The optimal homogeneous outcome appears to be a function of the number of competing stations $n$.

\section{A. Nash equilibria}

From the utility definition, it is evident that the strategies $\left\{(\boldsymbol{x}, 1), \boldsymbol{x} \in[0,1)^{n-1}\right\}$ are not Nash equilibria anymore. In fact, when one station employs a channel access probability equal to 1 , the AP collides with probability $p_{A P}=1$, thus resulting in a downlink throughput $S_{d}$ equal to 0 . Thus, the station is motivated to reduce its transmission probability to a value lower than 1 . Conversely, the strategies $\{(\boldsymbol{x}, 1,1), \boldsymbol{x} \in$ $\left.[0,1]^{n-2}\right\}$ are still Nash equilibria, because for a given station $i$ the utility function is fixed to 0 , regardless of its specific strategy $\tau_{i}$. We define the social utility as:

$$
J_{B I}^{S}=\min _{i=1, \cdots n} J_{B I}^{i}
$$

The price of anarchy is infinite also in this case. But, differently from the unidirectional scenario, we are going to show that there is also a NE with a good social utility, i.e. with a price of stability near to 1 .

The following remark will be useful to characterize Nash equilibria and Pareto optimality.

Remark 4.1: Consider a generic station $i$ and the collision probability $p_{i}$ suffered because of the other station strategies. For a given $p_{i} \in(0,1)$, by taking into account that $\tau_{A P}$ depends on $\tau_{i}$ and $p_{i}$ according to 2$), S_{d}\left(\tau_{i}\right)$ is a monotonic decreasing function of $\tau_{i}$, starting from $S_{d}^{i}(0)>0$, and $S_{u}^{i}\left(\tau_{i}\right)$ is a monotonic increasing function of $\tau_{i}$, starting from $S_{u}^{i}(0)=0$.

From the previous remark, we can state that for $p_{i} \neq 1$, the best response of player $i$ to $p_{i}$ is the access probability $\tau_{i}^{(b r)}$ such that $S_{u}^{i}\left(\tau_{i}^{(b r)}\right)=S_{d}^{i}\left(\tau_{i}^{(b r)}\right)$. It follows that $\tau_{i}^{(b r)}$ is the solution of the following implicit equation:

$$
\begin{aligned}
\tau_{i}^{(b r)} & =\frac{\tau_{A P}}{n-(n-1) \tau_{A P}}= \\
& =\frac{f\left(1-\left(1-p_{i}\right)\left(1-\tau_{i}^{(b r)}\right)\right)}{n-(n-1) f\left(1-\left(1-p_{i}\right)\left(1-\tau_{i}^{(b r)}\right)\right)}
\end{aligned}
$$

It can be shown that the previous equation has a single solution $\tau_{i}^{(b r)}$ in the range $(0,1)$, which can be numerically solved in a few fixed point iterations.

Let us consider the homogeneous outcome $\tilde{\tau}_{B I}=$ $\left(\tilde{\tau}_{B I}^{*}, \tilde{\tau}_{B I}, \cdots \tilde{\tau}_{B I}\right)$, such that

$$
\tilde{\tau}_{B I}=\frac{f\left(1-\left(1-\tilde{\tau}_{B I}\right)^{n}\right)}{n-(n-1) f\left(1-\left(1-\tilde{\tau}_{B I}\right)^{n}\right)} .
$$

We are going to show that $\tilde{\tau}_{B I}$ is a NE.

Proposition 4.1: The outcomes $\left\{(\boldsymbol{x}, 1,1), \boldsymbol{x} \in[0,1]^{n-2}\right\}$ and $\tilde{\tau}_{B I}$ are the unique Nash equilibria of the game.

Proof: We have already observed that the outcome in $\left\{(\boldsymbol{x}, 1,1), \boldsymbol{x} \in[0,1]^{n-2}\right\}$ are NE also in the bidirectional traffic scenario, while outcomes $\left\{(\boldsymbol{x}, 1), \boldsymbol{x} \in[0,1)^{n-1}\right\}$ are not. We can then restrict our analysis to the set $[0,1)^{n}$ for which $p_{i}<1$ for each $i$ and then best response satisfies (11).

$\tilde{\boldsymbol{\tau}}_{B I}$ is a NE because it satisfies equation (11) considering $p_{i}=\left(1-\tilde{\tau}_{B I}^{*}\right)^{n-1}$. Indeed the equation can be read as a mutual best response. Since equation (11) has a single solution, it exists a unique symmetric NE.

Now we need to prove that there is no asymmetric NE in $[0,1)^{n}$. Being that

$$
\left(1-p_{i}\right)\left(1-\tau_{i}\right)=\prod_{j=1}^{n}\left(1-\tau_{j}\right), \forall i
$$


then the right hand of the best response equation (11) is the same for all the stations. This excludes that a nonhomogeneous outcome can satisfy it and then be a NE.

We can consider which outcomes maximize the social utility and prove that

Proposition 4.2: There is a unique outcome that maximizes $J_{B I}^{S}$ and such outcome is homogeneous, i.e. $\tau_{B I}^{*}=$ $\left(\tau_{B I}^{*}, \tau_{B I}^{*}, \ldots \tau_{B I}^{*}\right)$, and Pareto optimal.

The proof is omitted because similar to that of proposition 3.2. It is interesting to investigate what is the relation between $\tau_{B I}^{*}$ and $\tilde{\tau}_{B I}$. For example Figure 2 shows how the utility changes for homogeneous outcomes and it seems that $\tau_{B I}^{*}=\tilde{\tau}_{B I}$ because the maximum of $J_{B I}^{i}\left(=J_{B I}^{S}\right.$ for a homogeneous case) corresponds to the value where $S_{u}^{i}=S_{d}^{i}$ as it is evident from the cusp in the curve. This happens when the point of maximum of $S_{u}^{i}((\tau, \tau, \cdots \tau))$ is for $\tau \geq \tau_{B I}^{*}$, so that $\tau_{B I}^{*}$ is the point of maximum of $J_{B I}^{S}$. Our numerical results show that this is the case at least for the realistic values we considered for the parameters in (2) $\left(C W_{\min }\right.$, $\left.C W_{\max }, R\right)$, but we were not able to prove that the result is general. We observe that when $\tau_{B I}^{*}=\tilde{\tau}_{B I}$ the NE $\tilde{\tau}_{B I}$ is also the best social outcome and the price of stability is then equal to 1 .

An interesting remark is that the NE $\tilde{\tau}_{B I}$ only depends on the number of stations $n$ and it is not affected either by the PHY layer parameters (such as backoff slot duration, interframe spaces, etc.) or by the frame length. This result is different from the optimal channel access probability derived in [8]. In fact, the result in [8] is based on the assumption that all stations use a fixed channel access probability, i.e. a fixed contention window. Conversely, in our scenario the AP adopts the standard exponential backoff scheme, thus adapting its contention window to the level of congestion perceived in the network. This adaptability allows the mobile stations to tune their optimal strategy regardless of the transmission times and backoff duration.

We verified via simulation that, starting from any strategy vector $\tau$, the station strategies converge to the NE $\tilde{\tau}_{B I}$ in a few steps, whenever strategy adjustments based on best response criteria are employed simultaneously by the stations.

\section{B. Mechanism Design}

We could argue that the system performance can be further improved by also tuning the AP contention window to a fixed value. In these conditions, since $\tau_{A P}$ does not depend on $\tau$ anymore, the best response for all the stations is equal to $\tau^{+}=\frac{\tau_{A P}}{n-(n-1) \tau_{A P}}$ and the $\mathrm{NE}$ equilibrium point in $(0,1)^{n}$ becomes $\left(\tau^{+}, \tau^{+}, \cdots \tau^{+}\right)$. By maximizing the $\mathrm{NE}$ equilibrium utility as a function of the parameter $\tau_{A P}$, it can be found a single optimal $\tau_{A P}^{*}$, that for $T \gg \sigma$ can be approximated as:

$$
\tau_{A P}^{*} \approx \frac{1}{\sqrt{2 T / \sigma}} .
$$

Note that the optimal $\tau_{A P}$ is not proportional to $1 / n$ as in (IV-B), because the AP downlink throughput corresponds to the aggregation of $n$ flows, whose bandwidths is equal to the uplink throughput perceived by each station at the NE point. Such a point corresponds to $\tau^{+}=\frac{1}{n \sqrt{2 T / \sigma}-(n-1)}$, which depends on both $n$ and the parameters $T$ and $\sigma$ as in [8].

We verified via simulation that the payoffs obtained for the NE $\tau$ with a legacy AP are about the same payoffs obtained with a fixed $\tau_{A P}$ value. Thus, since in the first case the equilibrium strategies do not depend on $T$ and $\sigma$, the use of a legacy AP is a simpler solution able to achieve high values of social utility.

\section{COnClusions}

In this paper we consider the role of the Access Point in infrastructure mode operation, for mitigating or discouraging potential selfish behaviors from the node. We have formulated some realistic games both for unidirectional and bidirectional (uplink and downlink) traffic scenarios and determined the set of Nash equilibria of these games. In the unidirectional scenario in absence of some punishment mechanisms, implemented at the AP side, nodes selfishness would lead to very poor performance. We identify adequate punishment mechanisms. On the contrary in the bidirectional scenario, a legacy Access Point is sufficient to achieve quasi-optimal performance without any need of a specific configuration.

\section{REFERENCES}

[1] IEEE Standard 802.11 - 1999; Wireless LAN Medium Access Control (MAC) and Physical Layer (PHY) Specifications; November 1999.

[2] Wi-Fi Alliance, www.wi-fi.org

[3] G. Bianchi, A. Di Stefano, C. Giaconia, L. Scalia, G. Terrazzino, I. Tinnirello, "Experimental assessment of the backoff behavior of commercial IEEE 802.11b network cards", Proc. of IEEE Infocom 2007, May 2007, Anchorage, pp. 1181-1189.

[4] S.W. Kim, B.S. Kim, Y. Fang, "Downlink and uplink resource allocation in IEEE 802.11 wireless LANs", Proc. of IEEE Conf. on Vehicular Technology, Jan. 2005, Dallas, vol. 54, pp. 320-327.

[5] M. Cagalj, S. Ganeriwal, I. Aad, J.P. Hubaux, "On selfish behavior in CSMA/CA networks", Proc. of IEEE Infocom, March 2005, Miami, vol. 4, pp. 2513-2524.

[6] L. Chen, S.H. Low, J. Doyle, "Contention Control: A Game-Theoretic Approach”, Proc. of IEEE Conf. On Decision and Control, Dec. 2007, New Orleans, pp. 3428-3434.

[7] G. Zhang, H. Zhang, "Modelling IEEE 802.11 DCF in wireless LANs as a dynamic game with incompletely information", Proc. of Conf. on Wireless, Mobile and Multimedia Networks, Jan. 2008, Mumbai, pp. 215-218.

[8] G. Bianchi, "Performance Analysis of the IEEE 802.11 Distributed Coordination Function", IEEE Journal of Selected Areas in Communication, vol. 18, no. 3, pp.535-547, March 2000.

[9] G. Bianchi, I. Tinnirello "Remarks on IEEE 802.11 Performance Analysis", IEEE Communication Letters, Vol. 9, no. 8, August 2005.

[10] I.Tinnirello, L. Giarré, G. Neglia, "The Role of Access Point against Selfish Nodes", INRIA Research Report, no. 6737, 2008 